\title{
Hospitalización por reacciones adversas a medicamentos
}

\author{
Mireya López-GamboA* \\ Centro Institucional de Farmacovigilancia, Instituto Nacional de Cancerología; Pro Pharma Research Organization, México
}

\begin{abstract}
RESUMEN
La relevancia de las reacciones adversas a medicamento (RAM) como causa de hospitalización de pacientes se está documentando cada vez más. Los países desarrollados están reportando un alto impacto en el incremento de costos de atención sanitaria por motivo de las RAM. La identificación de factores de riesgo para que una RAM cause hospitalización y la búsqueda de estrategias para contenerlas y así limitar la necesidad de hospitalización son un trabajo continuo. El objetivo de esta revisión fue documentar los principales factores de riesgo que causan hospitalización por RAM. Se encontró convergencia en que aquellos pacientes expuestos a polifarmacia y la población geriátrica tienen mayor riesgo de requerir hospitalización cuando se presentan reacciones adversas a medicamentos. Así mismo, múltiples autores están de acuerdo en que un porcentaje de estas RAM son prevenibles, para lo cual deben ser identificadas, evaluadas y contextualizadas. La identificación de las RAM que son prevenibles, así como de los factores que las originan que pueden ser modificables, es un área de oportunidad para disminuir la hospitalización por RAM, siendo un mecanismo de ahorro de recursos materiales y optimización de la atención médica en los sistemas de salud.
\end{abstract}

Palabras clave: Reacción adversa a medicamentos. Evento adverso. Hospitalización. Farmacovigilancia.

\section{ABSTRACT}

The relevance of adverse drug reactions (ADR) as a cause of hospitalization of patients is being more frequently documented. Developed countries are reporting a high impact of ADR on the increase of related health care costs. The identification of risk factors for an ADR causing hospitalization and the search for strategies to contain ADR and thus limit the need for hospitalization is a continuous work. The objective of this review was to document the main risk factors that cause hospitalization due to ADRs. Convergence was found in those patients exposed to polypharmacy, and geriatric patients were found to be at a higher risk of hospitalization when an ADR occurs. Likewise, multiple authors agree that a percentage of these ADRs may be avoided, for which they must be identified, evaluated and contextualized. The identification of preventable ADRs and the factors that cause ADR is an area of opportunity to

Correspondencia:

*Mireya López-Gamboa

E-mail: fv.incan@gmail.com
Date of reception:08-02-2019

Date of acceptance: 26-03-2019

DOI: 10.24875/HMCM.19000185 
reduce hospitalization due to ADR, being a mechanism for decreasing costs on material resources and optimizing medical attention in health care systems. (Hosp Med Clin Manag. 2019;12:28-39)

Corresponding author: Mireya López-Gamboa, fv.incan@gmail.com

Key words: Adverse drug reactions. Adverse event. Hospitalization. Admissions. Pharmacovigilance.

\section{INTRODUCCIÓN}

El uso de medicamentos busca causar en los pacientes efectos deseados como el alivio y control de signos y síntomas, e incluso la cura de enfermedades. Sin embargo, en todos los casos también existe la posibilidad de que el paciente presente signos o síntomas no deseados. En la literatura médica científica se han utilizado múltiples conceptos para describir estos efectos no deseados, como: efectos secundarios, reacciones adversas, efectos colaterales, eventos adversos, etc. Todos hacen referencia a signos o síntomas no deseados que un paciente presenta después de la administración de uno o más medicamentos; mundialmente, estos términos son utilizados para denominar las reacciones adversas a medicamentos (RAM); pueden verse alterados al ser traducidos, llegando a generar confusión en cuanto al concepto y su utilización. No obstante el término utilizado, el monitoreo permanente de éstos es indispensable para conocer el perfil de seguridad de los medicamentos ${ }^{1,2}$.

La normativa mexicana actual define como: a) Sospecha de Reacción Adversa a Medicamento (SRAM) a cualquier manifestación clínica o de laboratorio no deseada que ocurre después de la administración de uno o más medicamentos; b) Reacción Adversa a un Medicamento (RAM) a la respuesta no deseada a un medicamento, en la cual la relación causal con este es, al menos, razonablemente atribuible; c) Evento Adverso (EA) a cualquier suceso médico indeseable que pueda presentarse durante la etapa de investigación clínica de un medicamento pero que no necesariamente tiene una relación causal con este ${ }^{3}$. El monitoreo de la seguridad de medicamentos comercializados y usados en la práctica clínica cotidiana se realiza principalmente por reportes espontáneos de RAM ${ }^{1}$; las agencias regulatorias Food and Drug Administration [FDA], European Medicines Agency [EMA], Agencia Nacional de Vigilancia Sanitaria [ANVISA], Comisión Federal para la Protección contra Riesgos Sanitarios [COFEPRIS], etc.) lo han utilizado como fuente de información lo utilizan para otorgar, mantener, restringir o incluso cancelar la autorización de comercialización de medicamentos con el fin de proteger la salud pública. En México, la Secretaría de Salud, por medio de COFEPRIS, cumple con la Ley General de Salud², que indica que solo se concede autorización de comercialización a los medicamentos que demostraron reunir las características de calidad, seguridad y eficacia².

\section{EVALUACIONES RECIENTES DE LA INCIDENCIA DE HOSPITALIZACIÓN POR EVENTOS ADVERSOS, REACCIONES ADVERSAS A MEDICAMENTOS O SOSPECHA DE REACCIÓN ADVERSA A MEDICAMENTO}

En los últimos años, y principalmente en países desarrollados, se han realizado estudios prospectivos y retrospectivos para identificar la frecuencia, incidencia o prevalencia de las RAM en el proceso de hospitalización. Los resultados de los autores revisados confluyen en la relevancia de la identificación de factores predisponentes a las RAM, así como la posibilidad de prevenir las RAM y la hospitalización por RAM, ya que la incidencia de hospitalización asociada a SRAM o RAM está entre el 1 y el $47 \%$.

Un estudio realizado en los EE.UU. entre 2007 y 2009, sobre la base de 12,666 casos, reportó un promedio de 265,802 visitas anuales al Servicio de Urgencias, las cuales ocurrieron por EA de medicamentos; el 37.5\% de estas visitas $(99,628$ visitas) requirieron hospitalización (incluyendo internamiento [34.1\%], estancia en observación [2.2\%] y traslado a otro hospital [1.1\%]). Casi la mitad de las hospitalizaciones por EA por medicamentos (48.1\%; intervalo de confianza del 95\% [IC 95\%]: 44.6-51.6) ocurrieron en adultos de $\geq 80$ años de edad. La tasa poblacional de hospitalizaciones por EA por medicamentos fue 3.5 veces más alta entre los adultos de $\geq 85$ años de edad que entre los adultos de 65 a 69 años de edad (4.6 hospitalizaciones por 1,000 personas [IC 95\%: 2.5-6.8] comparado con 1.3 por 1,000 personas [IC 95\%: 0.7-1.8]) y se mantuvo significativamente elevada cuando se estratificó según el 
número de medicamentos concomitantes ( 0 a $40 \geq 5$ medicamentos concomitantes) $)^{4}$.

En EE.UU., entre 2006 y 2010, un estudio de cohorte retrospectivo en población infantil ( 0 a 18 años de edad) encontró que las enfermedades crónicas complejas (ECC) aumentaban el riesgo de visitas al Servicio de Urgencias relacionadas con RAM (de 144,385, 188 visitas, 716,664 visitas [0.5\%] fueron asociadas a RAM); $y$ que la presencia de una RAM aumentaba la probabilidad de ingreso hospitalario (tasa de probabilidad: 2.18; IC 95\%: 2.04-2.32), pero no encontró diferencia entre Ios niños con ECC o sin ECC (odds ratio [OR]: 0.93; IC: $0.84-1.03)^{5}$. En Brasil, en un estudio que evaluó 248 pacientes hospitalizados por más de 24 horas, se estimó en 46.4\% (115 de 248 pacientes) la prevalencia de hospitalización debido a EA de medicamentos ${ }^{6}$. En Francia, un estudio realizado para evaluar la incidencia de ingresos hospitalarios relacionados con RAM y la frecuencia de los que eran prevenibles, encontró que, de 2,692 hospitalizaciones, 97 se relacionaron con una RAM (incidencia: 3.6\%; IC 95\%: 2.8-4.4), destacándose que un tercio (32\%) de las hospitalizaciones relacionadas con RAM eran "prevenibles" y otro $16.5 \%$ «potencialmente prevenibles» ${ }^{7}$.

En Europa, las RAM son una causa considerable de morbilidad y mortalidad. Se ha estimado que aproximadamente el $5 \%$ de todos los ingresos hospitalarios son causados por RAM, que el $5 \%$ de los pacientes hospitalizados tendrán una RAM durante su estancia en el hospital, y que las RAM causan 197,000 muertes anuales en toda la Unión Europea ${ }^{8}$. Una revisión de 1,688 artículos publicados entre 2000 y 2014 seleccionó 104 textos completos y en 32 de estos artículos la hospitalización estuvo asociada a las RAM. En conjunto, un total de 110,427 pacientes se hospitalizaron o acudieron a urgencias por una RAM, lo cual representó el 3.6\% del total de admisiones hospitalarias (rango del 0.5 al $12.8 \%$ de todos los pacientes). Los estudios provenían de 12 países: Francia, Reino Unido, Alemania, Italia, Suiza, Grecia, España, Rumania, Eslovenia, Austria, Países Bajos y Noruega ${ }^{8}$. Así, un estudio observacional prospectivo en la población general adulta en Singapur evaluó a 1,000 pacientes ingresados a hospital durante un periodo de 3 meses para determinar la prevalencia de hospitalización relacionada con RAM. En 81 de estos pacientes la RAM fue la causa de ingreso hospitalario (8.1\%; IC 95\%: 6.5 -10.0\%; o 65\% de los pacientes que experimentaron una RAM), registrándose un total de 135
RAM en estos 124 pacientes. Dos tercios de todos los pacientes tomaban cinco o más medicamentos antes del ingreso. La mayoría de las RAM (91.9\%) tenían niveles de gravedad de 1 a 3 y posiblemente eran evitables (94.1\%) $)^{9}$. También, en España, del 2001 al 2010, un estudio determinó la prevalencia de admisiones debida a RAM y si la admisión era evitable. De los 3,292 pacientes con una edad media de 84.7 años, 197 pacientes fueron admitidos por una RAM $(5.98 \%$; IC 95\%: 5.17-6.79\%). De estas admisiones, 152 (76.4\%; IC 95\%: 70.4-82.4\%) se consideraron evitables ${ }^{10}$. Hace 4 años se publicó el metaanálisis de Oscanoa, et al., que abarcó desde 1988 hasta 2015; en un total de 42 artículos que reportaron ingresos hospitalarios provocados por RAM en pacientes mayores de 60 años, encontraron que el porcentaje general de ingresos fue del 8.7\%. Los datos del metaanálisis provenían de 21 países (América: EE.UU., Canadá, Brasil y Perú; Europa: Holanda, Italia, Bélgica, España, Francia, Reino Unido, Alemania, Austria, Eslovaquia, Grecia, Irlanda y Suecia; Asia: Taiwán, India y China; Oceanía: Australia; y África: Sudáfrica). El número de pacientes incluidos en cada estudio varió de 80 a 6,641,867 pacientes; en sus resultados encontraron que se reportaron entre 12 y 265,801 ingresos hospitalarios como consecuencia de alguna RAM, siendo EE.UU. el país con mayor número de admisiones hospitalarias. Los profesionales de la salud involucrados en la identificación de RAM fueron solo médicos en el $60 \%$ de los estudios, médicos y farmacéuticos en el $21 \%$, y médicos, farmacéuticos y enfermeras en el $19 \%$ de los estudios ${ }^{11}$.

Por otra parte, en Latinoamérica, Rojas, et al. realizaron un estudio para determinar si las RAM causaban ingresos a la unidad de cuidados intensivos ( $\mathrm{UCl}$ ) de un hospital universitario en Colombia. De 697 pacientes ingresados en la $\mathrm{UCl}, 96$ tenían al menos una RAM como su causa de ingreso (13.8\%; IC 95\%: 11.2-16.4\%), y en total se identificaron 108 casos con RAM (cada paciente tuvo entre una y tres RAM). El rango de edad de los pacientes fue de 17 a 94 años; sin embargo, el $77 \%$ tenía más de 60 años $^{12}$. En Brasil, un estudio prospectivo evaluó 472 pacientes tratados en un hospital de enseñanza entre 2010 y 2013, de los cuales 8 pacientes (1.7\%) fueron hospitalizados debido a RAM. La estancia media en el hospital fue de 16.8 días y el número promedio de medicamentos utilizados durante la hospitalización fue de 15.5 medicamentos por paciente. Se observaron 94 RAM en 75 pacientes, que tuvieron al menos una RAM ${ }^{13}$. 
Más recientemente, en Alemania, en el 2018, se determinó el porcentaje de casos con SRAM entre todos los pacientes que acudieron al Servicio de Urgencias durante un periodo de observación de 30 días, analizándose 10,174 casos. En estos se detectaron 665 SRAM, que corresponden al $6.5 \%$ de todos los $\operatorname{casos}^{14}$. Por otro lado, en Italia, se evaluaron 4,802 pacientes ingresados a hospitalización en los servicios de geriatría, medicina interna y enfermedades metabólicas durante 2 años para determinar el porcentaje de las RAM relacionadas con el ingreso hospitalario y sus características. En 296 pacientes las RAM fueron la causa de la hospitalización, lo que dio como resultado una prevalencia de ingreso hospitalario relacionado con RAM del 6.2\% (IC 95\%: 5.5-6.8); y también concluyeron que la mayoría de estas RAM podían ser prevenibles ${ }^{15}$. Por otra parte, un estudio de vigilancia prospectiva en los Emiratos Árabes Unidos evaluó la prevalencia de RAM debidas a medicamentos cardiovasculares en pacientes ingresados en un hospital de atención secundaria. De un total de 309 pacientes incluidos, 72 experimentaron 109 RAM, lo que representa una incidencia del 23.3\%; 12 pacientes fueron hospitalizados debido a RAM ( $n=16)$, mientras que 60 pacientes desarrollaron RAM ( $n=93$ ) durante su estancia hospitalaria. No hubo diferencia significativa en el número de RAM entre hombres y mujeres, $1.4 \pm 0.5$ y $1.6 \pm 0.9$, respectivamente ( $p=0.183$ ). El $59.54 \%$ de los pacientes eran mayores de 60 años y tuvieron un total de 73 RAM, con una tasa de incidencia del $24.5 \%$, mientras que un $40.45 \%$, que eran menores de 60 años, presentaron 36 RAM, con tasa de incidencia del $21.6 \%$, sin que esto fuera estadísticamente significativo $(p=0.834)^{16}$.

\section{IMPACTO DE LAS REACCIONES ADVERSAS A MEDICAMENTOS QUE CAUSAN HOSPITALIZACIÓN EN LA MORBILIDAD Y MORTALIDAD}

Las RAM son una causa importante de morbilidad y mortalidad, y llegan a ser la principal causa de ingreso hospitalario. Además una consecuencia de las RAM es la prolongación de la estancia hospitalaria, con el consecuente incremento del riesgo de morbilidad adquirida. Datos de la Organización Mundial de la Salud (OMS) sobre morbilidad hospitalaria en Europa estiman que cada año se hospitalizan 83.8 millones de pacientes en 31 países europeos, con una población combinada de 504 millones de personas. Una tasa de 0.5\% de RAM mortales podría significar que casi 419,000 personas mueren a causa de las RAM cada año en Europa. El uso de la tasa reportada de 0.05\% de RAM fatales ${ }^{17}$ da como resultado un estimado de 42,000 muertes debidas a RAM ${ }^{8}$. En el hospital Central de la universidad de Helsinki, la evaluación de 1511 muertes mostró que 75 casos fueron asociados con RAM (incidencia del 5\%) ${ }^{17}$. La evaluación de impacto en la mortalidad fue de relevancia para la generación de la nueva legislación de farmacovigilancia en Europa, donde se utilizó una estimación de 197,000 muertes al año, que se basó en la extrapolación de un estudio de los EE.UU. Esta estimación parece estar en un rango plausible basado en los estudios que se incluyeron en el estudio actual, y sugeriría que aproximadamente 0.25\% (o 1 de cada 400 pacientes hospitalizados) de todos los pacientes que no están hospitalizados debido a una RAM morirán como resultado de una RAM durante su estancia en un hospital europeo ${ }^{8}$.

La revisión de Bouvy, que partió de 47 artículos seleccionados, reportó ingresos hospitalarios debido a una RAM en 3.5\% (basado en 22 estudios) ${ }^{8}$. Uno de ellos mostró que el $5 \%$ de todas las muertes fueron causadas por una RAM y que el $0.05 \%$ de todas las hospitalizaciones dieron lugar a una RAM mortal en el 2016. La prevalencia de multimorbilidad (la coexistencia de enfermedades crónicas múltiples) en pacientes de edad avanzada se reportó entre el 55 y el 98\%; identificando a esta comorbilidad como un predictor de la hospitalización relacionada con RAM en pacientes de edad avanzada. Entre las comorbilidades relevantes se encontraron cardiopatía isquémica, insuficiencia cardíaca, depresión, diabetes, enfermedad vascular periférica, enfermedades pulmonares, reumatológicas, hepáticas, renales y malignas ${ }^{18}$.

Es pertinente mencionar que no en todos los artículos se han podido encontrar diferencias significativas entre la mortalidad asociada a hospitalizaciones por RAM y mortalidad de hospitalizaciones sin RAM. Como ejemplo está el estudio reportado por Chan, et al., quienes identificaron en 1,000 pacientes evaluados un total de 30 muertes; sin embargo, no hubo diferencias estadísticamente significativas en la tasa de mortalidad entre los pacientes con o sin RAM (3.4\% frente a 3.0\%, respectivamente; $p=0.781$ ); de los 4 pacientes con RAM asociada a la hospitalización que murieron, la causa de la muerte se relacionó con RAM en 2 de 
ellos$^{9}$. En el mismo sentido, Cabré, et al. refirieron no encontrar diferencias estadísticamente significativas entre la mortalidad intrahospitalaria asociada a admisiones por RAM frente a las admisiones sin RAM (8.0\% comparado con $8.2 \% ; p=0.919$ ). Los autores identificaron que, de las 16 muertes en el grupo de admisiones, 6 (37.5\%; IC 95\%: 13.9-61.1\%) se relacionaron directamente con una RAM. Por otro lado, a diferencia de lo reportado por otros autores, ellos no observaron diferencias significativas en la estancia hospitalaria para las admisiones de RAM frente a admisiones sin RAM (10.2 frente a 10.0 días; $p=0.715)^{10}$.

Como un aspecto de morbilidad, Wadhwa, et al. documentaron una estancia hospitalaria mayor en los pacientes que desarrollaron RAM (llegando hasta 10.11 días) que en los pacientes sin RAM (solo 6.29 días) ${ }^{16}$. En tanto, Giardina, et al. encontraron que los pacientes ingresados debido a RAM en hospitales de Italia tenían mayor afectación por hipertensión $(p<0.001)$, enfermedades cardíacas isquémicas ( $p=0.033)$, arritmia ( $p=0.002)$, enfermedades renales $(p<0.001)$ y enfermedad pulmonar obstructiva crónica $(p=0.029)$ que los pacientes sin RAM ${ }^{15}$. Estos resultados correlacionan con un estudio previamente publicado en 2013, el cual reportó aumento del promedio de estancia hospitalaria, de 8 días en pacientes sin RAM a 20 días en pacientes con RAM ${ }^{19}$.

Referente a la mortalidad asociada a RAM, un metaanálisis en EE.UU., en 1998, identificó a las RAM entre la cuarta y sexta causa de muerte, solo después de cardiopatía isquémica, cáncer y accidente cerebrovascular. Una década después, en la Unión Europea se identificaron a las RAM como causa de 197,000 muertes por año ${ }^{15}$. Otro metaanálisis publicado en el 2018 estimó la prevalencia de mortalidad entre los pacientes debido a las RAM que conducían a la hospitalización. Se exploró la heterogeneidad en su estimación mediante el análisis de subgrupos de las características del estudio y se identificaron los órganos y sistemas, así como medicamentos implicados y causantes de las RAM fatales. Entre los 49 estudios incluidos en el metaanálisis, 10,444 de 324,203 pacientes ingresaron debido a RAM y 419 pacientes murieron debido a una RAM. El número de muertes entre los estudios varió de 0 a 113. La prevalencia de reacciones fatales varió del 0 al $5.19 \%$. Diecinueve estudios reportaron cero muertes. La prevalencia media de RAM fatales fue del $0.20 \%$ (IC 95\%: 0.13-0.27\%; ; coeficiente de heterogeneidad $\left.\left[I^{2}\right]=93 \%\right)^{20}$.

\section{FACTORES DE RIESGO IDENTIFICADOS EN LA HOSPITALIZACIÓN POR REACCIONES ADVERSAS A MEDICAMENTOS}

Además de identificar las RAM que causan hospitalización, es sumamente relevante conocer cuáles son los factores asociados a que una RAM genere una hospitalización. Particularmente, la identificación de estos factores permite analizar cuáles de ellos son modificables, y así buscar estrategias para disminuir las hospitalizaciones asociadas a RAM. A continuación se describen los principales factores identificados en los artículos aquí revisados. En general se han clasificado como factores de riesgo para la aparición de RAM los siguientes: a) factores de riesgo relacionados con la medicación; b) relacionados con la enfermedad; c) relacionados con la capacidad funcional; d) relacionados con la adherencia a la medicación, y e) relacionados con el paciente ${ }^{21}$. Según Price, et al., la prescripción de medicamentos inapropiada se asoció con un aumento significativo en las hospitalizaciones no planificadas (OR: 1.18; IC 95\%: 1.15-1.21). Asimismo, basándose en un estudio de cohorte retrospectivo, se encontró un aumento en hospitalización (OR: 1.99; IC 95\%: 1.762.26) en pacientes de mayor edad que recibieron una prescripción inapropiada ${ }^{22}$. De los pocos reportes en población pediátrica, se encontró que pacientes con enfermedades crónicas complejas tuvieron un mayor riesgo de acudir al Servicio de Urgencias por RAM; los medicamentos asociados a las RAM con mayor frecuencia fueron agentes psicotrópicos, agentes antimicrobianos, anticonvulsivantes, hormonas/esteroides y analgésicos ${ }^{5}$.

La bibliografía mostró que las RAM representan un problema médico importante en geriatría, aun cuando los factores predictivos no se conocen bien en esta población. La edad se identificó como un factor significativo en la hospitalización relacionada con RAM. Las probabilidades de experimentar RAM severas aumentaron en un $3 \%$ por aumento de año de vida en personas mayores de 66 años. La cantidad de medicamentos concomitantes también se ha destacado en muchos estudios como un factor de riesgo independiente para los ingresos hospitalarios relacionados con RAM. Se ha estimado que la posibilidad que tiene un adulto mayor de presentar una RAM aumenta en un 10\% cuando se usa un medicamento y en un $75 \%$ si se usan más de cinco medicamentos al mismo tiempo. El aumento del riesgo de que un paciente geriátrico (edad media 70 años) 
tenga una hospitalización relacionada con una RAM fue del $24 \%$ por cada aumento de medicamento. Además, se ha documentado que la comorbilidad predice la admisión hospitalaria repetida por RAM en pacientes mayores. Finalmente, la prescripción de medicamentos potencialmente inapropiada, que es altamente prevalente en pacientes geriátricos ambulatorios, estuvo significativamente asociada con RAM y la posterior admisión hospitalaria ${ }^{18}$. También, el estudio de Rojas, et al. identificó la polifarmacia como un factor de riesgo relevante, ya que los pacientes hospitalizados refirieron que tomaban entre uno y 16 medicamentos de forma concomitante $^{12}$. En este mismo sentido, de 352 de pacientes con casos de SRAM en el Servicio de Emergencias con edades de 18 a 97 años, el 75\% tenía 65 años o más ${ }^{14}$. Además, se observó que, a mayor cantidad de medicamentos, mayor posibilidad de encontrarse una RAM, y que los pacientes con RAM recibían concomitantemente un promedio de siete diferentes medicamentos (rango: 1-18 fármacos o sustancias activas); conjuntando ambos factores, el estudio determinó que el número de medicamentos concomitantes aumentaba con la edad de los pacientes ${ }^{14}$.

Entre otros factores identificados como predictivos para presentar alguna RAM que requirió hospitalización, se encontró el antecedente de que el paciente utilizara alguno de los medicamentos identificados lista de Base de conocimiento farmacogenómico (PharmGKB), anotación clínica (CA ), lo cual refleja la relevancia del conocimiento farmacogenético ${ }^{9}$. En 28,411 pacientes, se encontraron 964 casos de hospitalización de los que se reportó la presencia de interacciones farmacológicas y el género como los principales factores de riesgo ${ }^{23}$; sin embargo, basado en otro estudio de corte transversal, no se encontró que el sexo fuera un factor de riesgo ${ }^{18}$, por lo que la participación de estos factores, aun cuando tiene sentido, no ha sido confirmada por medio de datos estadísticos de la población.

\section{IMPACTO ECONÓMICO DE LAS RAM}

Además de los aspectos de deterioro de la salud, un factor importante a evaluar recientemente es el impacto económico que las RAM pueden tener en los sistemas de atención de salud. Si bien la metodología para evaluar los costos, así como la moneda en que se hace la evaluación, pueden diferir, cada vez se encuentra más bibliografía que documenta el impacto económico que los eventos y reacciones adversas representan para los sistemas de salud. Si bien, la exploración de la bibliografía para esta revisión buscó documentar la presencia y los factores de riesgo que llevan a hospitalizaciones por RAM; en el proceso se identificó que algunos de los estudios y revisiones consultadas abordan el impacto económico en sus análisis. Por la relevancia del impacto económico que la bibliografía revisada mostró, a continuación se presenta un resumen de los resultados de aquellos artículos que incluyeron evaluaciones económicas.

Para 2010 se había estimado que las RAM costaron aproximadamente 55 billones de dólares (USD) en Colombia ${ }^{12}$. Entre 2003 y 2008, Wu, et al. reportaron la incidencia y los costos de las visitas al Servicio de Urgencias relacionadas con RAM y los ingresos hospitalarios posteriores a la visita, en adultos de 66 años o más. Los costos estandarizados al 2008 e informados en dólares canadienses (CAD) mostraron lo siguiente: el costo promedio para cada paciente individual se mantuvo estable en el periodo evaluado; la atención médica de 7,222 pacientes con RAM que visitaron el Servicio de Urgencias costó un total de \$2,408,325 CAD; de ellos, en 5,660 pacientes con RAM leves el costo fue de $\$ 235$ CAD por persona por una visita, llegando a \$691 CAD cuando las RAM fueron severas. Además, al ser ingresados al hospital, los pacientes con RAM severas costaron un promedio de $\$ 7,529$ CAD por persona durante su estancia en el hospital. Como resultado global en 2007, el costo total medido de visitas al Servicio de Urgencias por RAM y las hospitalizaciones posteriores ascendió a \$13.6 millones de dólares canadienses $^{24}$. En este mismo sentido, la publicación de Nivya, et al., con datos de 15 estudios en países europeos, EE.UU., Canadá e India, concluye que a mayor gravedad de la RAM mayor costo, reportando un costo promedio de $\$ 342$ para las RAM de grado 4, el cual aumenta a $\$ 438$ para las de grado 5 y llega hasta $\$ 726$ para las de grado $6^{25}$.

El impacto y la gestión de las RAM son complejos; en EE.UU. pueden costar hasta 30.1 mil millones USD anuales. Se sabe que el incremento de los costos por RAM se relaciona con el aumento de hospitalizaciones, la prolongación de la estancia hospitalaria y los análisis de laboratorio adicionales requeridos para los casos graves. Además, las RAM pueden desencadenar cascadas de nuevas prescripciones para tratar las 
mismas RAM; esto aumenta los costos de la farmacoterapia y aumenta el riesgo de nuevas RAM $^{19}$. Un dato que destacar es que de las RAM que requieren hospitalización, el costo por RAM prevenibles es mayor que para las RAM no prevenibles. Se ha documentado también que el costo por RAM es de \$2,262 USD, y que pueden variar entre los servicios dentro del hospital, siendo de $\$ 13,994$ USD en áreas de cuidados no intensivos y alcanzando $\$ 19,685$ USD en las unidades de cuidados intensivos. Es relevante mencionar que los estudios de vigilancia de medicamentos identificaron que la fiebre, las hemorragias, la diarrea y las arritmias cardíacas, en orden decreciente, fueron las RAM que originaron la mayor carga económica en el ámbito hospitalario ${ }^{19}$.

Identificados por grupos de medicamentos, se encontró que los antiinflamatorios no esteroideos (AINE), antibióticos, anticoagulantes y agentes antineoplásicos son una causa importante de los costos relacionados con la RAM. Además de los costos financieros directos, también hay varios costos indirectos, poco evaluados, en los que incurren los pacientes y sus cuidadores por efecto de las RAM como, por ejemplo, los días de trabajo perdidos ${ }^{19}$.

Un estudio más reciente en pacientes de EE.UU. ha reportado los costos incrementales de atención médica asociados con EA de pacientes oncológicos bajo tratamiento. Cuando no se consideró la gravedad del evento adverso, los costos oscilaron entre $\$ 546$ USD para tos/infecciones respiratorias superiores y hasta $\$ 24,633$ USD para perforación gastrointestinal. Sin embargo, cuando el evento fue grave el costo se incrementó, oscilando entre $\$ 15,709$ USD para dermatitis y erupción cutánea, hasta $\$ 48,538$ para fístula gastrointestinal; así como \$24,322 USD para la hemorragia del sistema nervioso central y $\$ 23,510$ USD para sepsis/septicemia. Los autores concluyeron que las reacciones adversas durante los episodios de tratamiento para el cáncer fueron frecuentes y se asociaron con una carga económica sustancial26.

Aun cuando estos datos no describen todos los estudios farmacoeconómicos, son un referente del impacto negativo que pueden tener las RAM que causan hospitalización, y también permiten comprender que el manejo de las RAM prevenibles puede generar ahorros en los sistemas de salud y en la economía del propio paciente.

\section{HERRAMIENTAS PARA CLASIFICAR Y EVALUAR LOS EVENTOS ADVERSOS, LAS REACCIONES ADVERSAS A MEDICAMENTOS Y LAS SOSPECHAS DE REACCIÓN ADVERSA A MEDICAMENTO}

La necesidad de una evaluación estandarizada de las RAM es una prioridad motivada por el impacto negativo que tienen en la salud y en el incremento de los costos de la atención hospitalaria, así como su potencial de prevención. En general, cada una de las RAM se puede evaluar y clasificar en cuanto a diferentes parámetros. Los aspectos más comúnmente evaluados y descritos son la severidad de cada una de las RAM, la gravedad que el caso tiene al momento de presentarse la(s) RAM, así como el análisis de causalidad (por método de algoritmos o de criterios preestablecidos). Este último tiene como objetivo identificar la relación causa-efecto de la asociación medicamento-RAM, para así encontrar posibles estrategias de prevención o control de RAM. Uno de los métodos más utilizados es el algoritmo de Naran$\mathrm{jo}^{27}$, que consta de 10 preguntas con respuestas «sí/no/ no se sabe" y permite establecer una causalidad probabilística.

A pesar de su relevancia, los criterios para las evaluaciones de severidad, gravedad y causalidad utilizados en el mundo han sido diferentes, lo cual fue identificado en los artículos aquí mostrados. Esta limitante puede afectar la comparabilidad de los resultados entre los artículos, lo que se puede apreciar en la descripción de los análisis de causalidad en los artículos revisados, que mostramos a continuación. En su publicación, Varallo, et al. encontraron que los pacientes reportaron 178 RAM, 6 (3.4\%) de las cuales se clasificaron como definitivas, 54 (30.3\%) como probables y 118 (66.3\%) como posibles ${ }^{6}$. En la revisión de Bouvy, et al. encontraron que 32 de los 47 estudios incluidos evaluaron la causalidad de las RAM. Esta evaluación reportó el uso del algoritmo de Naranjo en 18 estudios, y el uso de los criterios de la OMS en 6 estudios, así como el uso del método de Karch y Lasagna en 2 estudios y otros métodos en los restantes 6 estudios. Sin embargo, solo 14 artículos reportaron la distribución de la evaluación de causalidad entre todas las RAM, lo que limitó la comparabilidad de estas ${ }^{8}$. En la publicación de Rojas, et al. se clasificaron las RAM para causalidad según el algoritmo de Naranjo, reportando como posible el $42.5 \%$ de los casos, probable el $32.4 \%$ y definitivo el $0.9 \%$; así mismo, reportaron 22 casos de falla terapéutica que 
correspondieron al $20 \%$ de todos los casos. Para la identificación de cuáles RAM fueron prevenibles utilizó los seis tipos propuestos por Edwards y Aronson en 2000, reportando 75 RAM del tipo A (69\%), 2 del tipo B (2\%), 5 del tipo C (5\%), 4 del tipo E (4\%) y 22 del tipo $F(20 \%)$. No se identificaron RAM de tipo $D^{12}$.

Por otra parte, Cabré, et al. definieron una RAM como cualquier efecto dañino no deseado de un medicamento que se produjo a niveles de dosis normales para la profilaxis, con fines de diagnóstico y tratamiento. No clasificaron como RAM las sobredosis deliberadas. Para decidir si una hospitalización debía clasificarse como relacionada con la RAM, usaron los criterios de probabilidad de la OMS-Centro de Monitoreo de Uppsala (UMC, por sus siglas en inglés). Para evaluar la probabilidad de que la RAM se produjera por un medicamento, utilizaron el algoritmo de Naranjo. La evitabilidad de una RAM fue categorizada según los criterios de Hallas, et al. (categorías: definitivamente evitable, posiblemente evitable o inevitable) $)^{28}$. Los resultados de revisar 3,292 pacientes arrojaron que 197 fueron hospitalizados por RAM (6\% de los ingresos) y que el $75 \%$ de estas RAM eran evitables. Entre las causas directas del ingreso estuvieron la toxicidad por digitálicos, insuficiencia renal aguda, deterioro cognitivo e hiponatremia $^{10}$. De una revisión de 10,174 casos de pacientes tratados, la evaluación de causalidad de la RAM se estandarizó según los criterios de la OMS-UMC, identificándose a cada caso de sospecha de RAM como posible, probable o seguro después de la evaluación. Solo para 350 de los casos se obtuvo el consentimiento del paciente para el uso de los datos; por lo que el análisis de causalidad reportó como posibles a 287 casos, probables a 57 casos y certeros a $8 \operatorname{casos}^{14}$.

Como se ha mencionado anteriormente, mejorar la evaluación de las RAM y buscar métodos para estandarizar sus escalas de evaluación es una necesidad cada vez más evidente. Esto pudimos observarlo en publicaciones recientes, donde se encontró un incremento en el uso y variedad de los métodos de evaluación y caracterización de las RAM. Así, Giardina, et al. reportaron el análisis de causalidad por el algoritmo de Naranjo; de un total de 4,802 pacientes evaluados, solo los casos identificados como certeros, posibles o probables fueron considerados en el análisis. Todas las RAM se codificaron según el Diccionario Médico para Actividades Regulatorias (MedDRA, por sus siglas en inglés) ${ }^{29}$, organizándolos de acuerdo con la clasificación por órganos y sistemas (SOC, System Organ Class) y el término preferido (PT, Preferred Term). También se realizó la clasificación de gravedad (se consideró grave cuando fue fatal, fue necesaria una hospitalización prolongada, fue potencialmente mortal o causó una discapacidad grave o permanente, o anomalía congénita/defecto de nacimiento $)^{30}$. La evitabilidad de la RAM se evaluó de acuerdo con los criterios de Schumock y Thornton. El diagnóstico de ingreso y alta hospitalaria y las enfermedades concurrentes se codificaron de acuerdo con la Clasificación Internacional de Enfermedades, 9. ${ }^{a}$ ed., Modificación Clínica (CIE-9-CM). Las comorbilidades se evaluaron mediante la puntuación de Charlson 15,31,32. En este mismo sentido, en el artículo de Riveiro, et al., de los 472 pacientes seleccionados, a todos aquellos con sospecha de RAM se les evaluó con el algoritmo de Naranjo $^{13}$. Se diferenciaron las RAM prevenibles de las no prevenibles según los criterios desarrollados por Rawlins y Thompson; se calificaron según la severidad (es decir, leve, moderada o severa) utilizando los criterios de Hartwig, et al. ${ }^{13,33}$

\section{REACCIONES ADVERSAS A MEDICAMENTOS MÁS FRECUENTES}

Como se ha ido mostrando en esta revisión, las RAM se han posicionado como una de las grandes preocupaciones que los profesionales de la salud y los sistemas sanitarios tienen en la actualidad. Por esto es importante conocer cuáles son las reacciones que más frecuentemente se presentan como consecuencia adversa del uso de medicamentos. En la tabla 1 se presentan los listados de las RAM y medicamentos asociados a las RAM descritos en los artículos revisados.

Para la unificación de la terminología utilizada para describir las RAM, cada vez más se utiliza la codificación MedDRA, la cual permite, al menos, identificar un PT y un SOC. Ambos son términos consensuados mundialmente que evitan la dilución al contabilizar los resultados por uso de términos diversos con mismo significado. Para los artículos revisados y mostrados en la tabla 1 se ha mantenido la terminología con que cada uno de ellos reportó las RAM. Además, dependiendo de la disponibilidad de los datos y el formato de reporte en los artículos respectivos, la tabla muestra en forma porcentual o como número absoluto las variables de interés: hospitalización RAM y medicamentos. 
Tabla 1. Listado de las reacciones adversas a medicamentos descritas en los artículos revisados

\begin{tabular}{|c|c|c|c|c|}
\hline $\begin{array}{l}\mathrm{N} .{ }^{0} \text { de casos } \\
\text { o pacientes } \\
\text { evaluados }\end{array}$ & $\begin{array}{l}\text { Hospitalizaciones } \\
\%\left(\text { n. }^{0}\right)\end{array}$ & $\begin{array}{l}\text { N. }{ }^{0} \text { de RAM } \\
\text { identificadas }\end{array}$ & $\begin{array}{l}\text { PT, SOC o RAM reportados en más del } \\
10 \% \text { (expresado como n. }{ }^{0} 0 \% \text { ) }\end{array}$ & $\begin{array}{l}\text { Medicamentos asociados a RAM en más } \\
\text { del } 10 \% \text { (expresado como n. }{ }^{\circ} 0 \% \text { ) }\end{array}$ \\
\hline $\begin{array}{l}\text { De } 10,174 \text { casos } \\
\text { revisados }^{14}\end{array}$ & $43.70 \%$ & 665 RAM & $\begin{array}{l}\text { T. sistema nervioso }(33 \%) \text {, t. } \\
\text { gastrointestinales }(32 \%) \text {, t. generales y } \\
\text { condiciones del sitio de administración } \\
(30 \%) \text {, t. respiratorios, torácicos y } \\
\text { mediastínicos }(18 \%) \text {, t. vasculares }(15 \%) \text {, } \\
\text { t. cardíacos }(13 \%) \text {, lesiones, } \\
\text { intoxicaciones } \\
\text { y complicaciones procesales }(15 \%), \text { t. } \\
\text { metabolismo y de la nutrición }(11 \%) \\
\text { Nota: Porcentajes aproximados tomados } \\
\text { de la gráfica del artículo }\end{array}$ & $\begin{array}{l}\text { Inhibidores de la ECA y bloqueadores del } \\
\text { receptor AT1 (17\%), antidepresivos (11\%), } \\
\text { agentes antineoplásicos e } \\
\text { inmunomoduladores (10\%), agentes } \\
\text { antitrombóticos }(26 \%) \text { betabloqueadores } \\
(18 \%) \text {, diuréticos }(13 \%) \text {, analgésicos } \\
\text { periféricos }(10 \%)\end{array}$ \\
\hline $\begin{array}{l}\text { De } 309 \\
\text { pacientes }^{16}\end{array}$ & $\begin{array}{l}3.88 \% \\
(12 \text { pacientes) }\end{array}$ & 109 RAM & $\begin{array}{l}\text { Bradicardia, hipotensión, creatinina sérica } \\
\text { elevada, desequilibrio electrolítico, } \\
\text { estreñimiento, INR elevado, malestar } \\
\text { abdominal, vómito, taquicardia, tos seca, } \\
\text { enzimas hepáticas elevadas, dolor de } \\
\text { cabeza, hemorragia gastrointestinal, edema } \\
\text { del pedal }\end{array}$ & $\begin{array}{l}\text { Bisoprolol }(16 ; 14.68 \%) \text {, amlodipino } \\
(10 ; 9.17 \%) \text {, warfarina }(9 ; 8.26 \%) \text {, } \\
\text { furosemida }(8 ; 7.34 \%) \text {, nitroglicerina } \\
(8 ; 7.34 \%) \text {, atorvastatina }(7 ; 6.42 \%) \text { y } \\
\text { aspirina }(7 ; 6.42 \%)\end{array}$ \\
\hline $\begin{array}{l}\text { De } 472 \\
\text { pacientes }^{13}\end{array}$ & $\begin{array}{l}1.7 \% \\
\text { (8 pacientes) }\end{array}$ & 94 RAM & $\begin{array}{l}\text { T. cardiovasculares (4), infecciones (3), t. } \\
\text { neurológicos (12), t. renales (17), t. de la } \\
\text { piel (10), t. sistémicos (1), t. endocrinos y } \\
\text { metabólicos (10), otros (2) }\end{array}$ & $\begin{array}{l}\text { Codeína, vancomicina, morfina, } \\
\text { metilprednisolona }\end{array}$ \\
\hline $\begin{array}{l}\text { De } 4,802 \\
\text { hospitalizaciones }^{15}\end{array}$ & $6.2 \%$ * & 310 RAM & $\begin{array}{l}\text { T. de la sangre y del sistema nervioso } \\
(27 \%) \text {, t. gastrointestinales }(28 \%) \text {, t. } \\
\text { metabolismo y nutrición }(18 \%) \text {, t. sistema } \\
\text { nervioso }(17 \%)\end{array}$ & $\begin{array}{l}\text { Antitrombóticos (39\%), inhibidores de RAS } \\
(14 \%) \text {, AINE }(10 \%)\end{array}$ \\
\hline $\begin{array}{l}\text { De } 49 \text { estudios } \\
\text { que evaluaron } \\
324,203 \\
\text { pacientes }^{20}\end{array}$ & 10,444 pacientes* & $\begin{array}{l}243 \text { RAM } \\
\text { fatales }^{\dagger}\end{array}$ & $\begin{array}{l}\text { T. sistema nervioso (91), t. } \\
\text { gastrointestinales (27), t. renales y } \\
\text { urinarios (26), t. metabolismo y nutrición } \\
\text { (15), t. vasculares (15), t. hepatobiliares } \\
\text { (11), t. sanguíneos y del sistema linfático } \\
\text { (6), t. cardíacos (5), t. respiratorios, } \\
\text { torácicos y mediastínicos (5), t. de la piel y } \\
\text { del tejido subcutáneo (2), lesiones, } \\
\text { intoxicaciones y complicaciones procesales } \\
\text { (2), t. psiquiátricos (1), infecciones e } \\
\text { infestaciones (1), reacciones no } \\
\text { especificadas (30) }\end{array}$ & $\begin{array}{l}\text { Antagonistas de la vitamina K/warfarina } \\
\text { (79), aspirina (11), aspirina + diclofenaco/ } \\
\text { rofecoxib /meloxicam/fluoxetina /warfarina } \\
\text { (5), inhibidores de RAS (15), diuréticos (8), } \\
\text { heparina + acenocoumarol + fluindiona + } \\
\text { ticlopidina (4), medicamentos } \\
\text { antituberculosos (9), medicamentos } \\
\text { anticancerígenos (2), digoxina + } \\
\text { furosemida/venlafaxina (2), tamoxifeno (1), } \\
\text { ampicilina + cotrimoxazol + sulfadoxina + } \\
\text { pirimetamina + ibuprofeno (1), ampicilina + } \\
\text { cotrimoxazol + sulfadoxina/pirimetamina } \\
\text { (1), lorazepam + zolpidem + codeína (1), } \\
\text { metilprednisolona + tacrolimús + } \\
\text { micofenolato (1), digoxina (14), } \\
\text { medicamentos inmunosupresores (5) }\end{array}$ \\
\hline $\begin{array}{l}\text { De } 697 \\
\text { pacientes }^{12}\end{array}$ & $\begin{array}{l}96 \\
\text { pacientes }\end{array}$ & 108 RAM & $\begin{array}{l}\text { Arritmia }(12 \%) \text {, sangrado gastrointestinal } \\
\text { superior }(12 \%) \text {, convulsiones }(11 \%) \text {, } \\
\text { hiperpotasemia }(9 \%) \text {, hipertensión ( } 8 \%) \text {, } \\
\text { enfermedad hemorrágica cerebrovascular } \\
(7 \%)\end{array}$ & $\begin{array}{l}\text { Metoprolol, clonidina, carvedilol, ácido } \\
\text { acetilsalicílico, ácido valproico, } \\
\text { carbamazepina, fenitoína losartán, warfarina, } \\
\text { hidroclorotiazida }\end{array}$ \\
\hline $\begin{array}{l}\text { De } 3,292 \\
\text { pacientes }^{10}\end{array}$ & 197 pacientes* & 197 RAM & $\begin{array}{l}\text { Toxicidad digitálica }(20.3 \%) \text {, delirium y } \\
\text { cambios en el estado mental relacionado } \\
\text { con opioides y consumo de medicamentos } \\
\text { psicotrópicas (10.1\%), insuficiencia renal } \\
\text { aguda relacionada con diuréticos }(5.6 \%), \\
\text { caídas relacionadas con benzodiacepinas } \\
(6.6 \%)\end{array}$ & $\begin{array}{l}\text { Digoxina }(20.3 \%) \\
\text { AINE }(13.2 \%) \\
\text { Benzodiacepinas }(10.6 \%) \\
\text { Diuréticos }(8.6 \%) \\
\text { Antibióticos }(4.6 \%)\end{array}$ \\
\hline
\end{tabular}


Tabla 1. Listado de las reacciones adversas a medicamentos descritas en los artículos revisados (continuación)

\begin{tabular}{|c|c|c|c|c|}
\hline $\begin{array}{l}N{ }^{0} \text { de casos } \\
\text { o pacientes } \\
\text { evaluados }\end{array}$ & $\begin{array}{l}\text { Hospitalizaciones } \\
\%\left(\mathrm{n} .{ }^{\circ}\right)\end{array}$ & $\begin{array}{l}\text { N. }{ }^{0} \text { de RAM } \\
\text { identificadas }\end{array}$ & $\begin{array}{l}\text { PT, SOC o RAM reportados en más del } \\
10 \% \text { (expresado como n. }{ }^{\circ} 0 \% \text { ) }\end{array}$ & $\begin{array}{l}\text { Medicamentos asociados a RAM en má } \\
\text { del } 10 \% \text { (expresado como n. }{ }^{0} 0 \% \text { ) }\end{array}$ \\
\hline
\end{tabular}

\begin{tabular}{|c|c|c|c|c|}
\hline $\begin{array}{l}\text { De } 42 \text { estudios, } \\
\text { cada uno reportó } \\
\text { de } \\
80 \text { a } 6,641 \\
867 \text { pacientes }^{11}\end{array}$ & $\begin{array}{l}\text { Rango reportado: } \\
12 \text { a } 265,801 \\
\text { pacientes }^{\ddagger}\end{array}$ & $\begin{array}{l}\text { Dato del } \\
\text { consolidado } \\
\text { no reportado }\end{array}$ & $\begin{array}{l}\text { AINE: } \\
\text { Anemia, úlcera gástrica, insuficiencia renal } \\
\text { aguda, hipersensibilidad, pancitopenia, } \\
\text { sangrado gastrointestinal, dolor epigástrico, } \\
\text { irritación estomacal, hipertensión, ulcera } \\
\text { péptica, crisis hipertensiva, hepatitis, } \\
\text { erupción cutánea, disnea } \\
\text { Betabloqueadores: } \\
\text { Síncope, bradicardia, caída, arritmias } \\
\text { cardíacas, hipotensión postural, fatiga, }\end{array}$ & $\begin{array}{l}\text { Betabloqueadores }(1.8-66.7 \%) \\
\text { Antibióticos }(1.1-22.2 \%) \\
\text { Anticoagulantes orales }(3.3-55.6 \%) \\
\text { Digoxina (1.6-18.8\%) } \\
\text { Inhibidores de ACE }(5.5-23.4 \%) \\
\text { Medicamentos anticancerígenos }(1.5-9.1 \%) \\
\text { Bloqueadores de entrada de calcio } \\
(1.0-8.3 \%) \\
\text { Opioides }(1.5-18.8 \%) \\
\text { Antidiabéticos orales }(4.5-22.2 \%) .\end{array}$ \\
\hline
\end{tabular}

Antibióticos:

Diarrea, vómito, insuficiencia renal, hipertensión, alegría, urticaria, síndrome de Stevens-Johnson, erupción cutánea Warfarina:

Anemia, sangrado gastrointestinal, INR alto, disnea, coagulopatía Digoxina:

Bradicardia, arritmias cardíacas, náusea, vómito, síncope, náusea, vómito, anorexia, delirium, falla cardíaca, bradicardia, hipotensión Inhibidores de ACE:

Hiponatremia, hiperpotasemia, síncope, insuficiencia renal, hipotensión, caída, hipercalemia, neutropenia Antitumorales:

Anemia, caída, pancitopenia, fiebre, diarrea, vómito, erupción en la piel

Bloqueadores de entrada de calcio: Síncope, bradicardia, caída, hipotensión, arritmias cardíacas, edema, síncope, vértigo, factura

Opioides:

Disturbios gastrointestinales, confusión, somnolencia, vértigo, fractura, cambio del estado mental

Antidiabéticos orales:

Hipoglucemia, acidosis, fatiga, estado mental alterado, fatiga

$\begin{array}{ll}\text { De } 1,000 & 124 \text { pacientes } \\ \text { pacientes }^{9} & \text { hospitalizados con } \\ & \text { RAM }\end{array}$

De 15 estudios de 49; rango de 683 a 11 millones pacientes $^{25}$
Rango de 683 a 11 Dato del millones de pacientes consolidado no reportado
135 RAM

Tracto gastro intestinal (25), anormalidades electrolíticas (23)

Hemorragias/INR elevado (20), empeoramiento renal (13), anemia/ leucopenia/ trombocitopenia (8), infección/ sepsis (6), hipotensión (6)

Neutropenia febril, desequilibrio electrolítico, alteración de INR, agranulocitosis, defectos de coagulación, insuficiencia cardíaca, insuficiencia respiratoria, problemas cardiovasculares, agranulocitosis, sangrado gastrointestinal, constipación, osteoporosis, hipoglucemia, anemia, constipación, embolia pulmonar, nefropatía
Medicamentos cardiovasculares (44) Medicamentos para t. sanguíneos (24), antineoplásicos inmunomoduladores (22), medicamentos del sistema nerviosos central (15)

Antineoplásicos e inmunomoduladores; vacunas, antiinfecciosos para uso sistémico; medicamentos para el sistema nervioso; diuréticos, agentes antitrombóticos e inhibidores de la ECA; terapia hormonal, antineoplásicos; inmunosupresores; antineoplásicos, antirreumáticos, anticoagulantes y salicilatos, esteroides y antibióticos 
Tabla 1. Listado de las reacciones adversas a medicamentos descritas en los artículos revisados (continuación)

\begin{tabular}{|c|c|c|c|c|}
\hline $\begin{array}{l}\mathrm{N} .{ }^{0} \text { de casos } \\
\text { o pacientes } \\
\text { evaluados }\end{array}$ & $\begin{array}{l}\text { Hospitalizaciones } \\
\%\left(\mathrm{n}^{0}\right)\end{array}$ & $\begin{array}{l}\text { N. }{ }^{0} \text { de RAM } \\
\text { identificadas }\end{array}$ & $\begin{array}{l}\text { PT, SOC o RAM reportados en más del } \\
10 \% \text { (expresado como n. }{ }^{0} \% \text { ) }\end{array}$ & $\begin{array}{l}\text { Medicamentos asociados a RAM en más } \\
\text { del } 10 \% \text { (expresado como n. }{ }^{\circ} 0 \% \text { ) }\end{array}$ \\
\hline $\begin{array}{l}\text { De } 2,692 \\
\text { pacientes }^{7}\end{array}$ & $\begin{array}{l}3.6 \%{ }^{\star} \\
\text { ( } 97 \text { pacientes) }\end{array}$ & $\begin{array}{l}\text { Dato del } \\
\text { consolidado } \\
\text { no reportado }\end{array}$ & $\begin{array}{l}\text { T. musculares }(20.6 \%) \\
\text { T. sistema nervioso central }(11.3 \%) \\
\text { T. gastrointestinales }(9.3 \%) \\
\text { T. generales }(9.3 \%)\end{array}$ & $\begin{array}{l}\text { Antitrombóticos }(12.6 \%) \\
\text { Antineoplásicos }(12.6 \%) \\
\text { Diuréticos }(9 \%) \\
\text { Analgésicos }(9 \%)\end{array}$ \\
\hline $\begin{array}{l}\text { De } 248 \\
\text { pacientes }^{6}\end{array}$ & $46.40 \%$ & 178 RAM & $\begin{array}{l}\text { Disnea }(15.2 \%) \text {, fatiga }(12.3 \%) \text {, dolor de } \\
\text { pecho }(9.0 \%) \text {, dolor abdominal }(8.9 \%) \text { e } \\
\text { hiperglucemia }(5.6 \%)\end{array}$ & $\begin{array}{l}\text { Omeprazol, captopril, insulina, ácido } \\
\text { acetilsalicílico, furosemida, propranolol, } \\
\text { simvastatina, carvedilol, digoxina, enalapril }\end{array}$ \\
\hline 12,666 casos $^{4}$ & $\begin{array}{l}5,077 \\
\text { hospitalizaciones } \\
7,589 \text { visitas } \\
\text { urgencias }\end{array}$ & $\begin{array}{l}\text { Dato del } \\
\text { consolidado } \\
\text { no reportado }\end{array}$ & 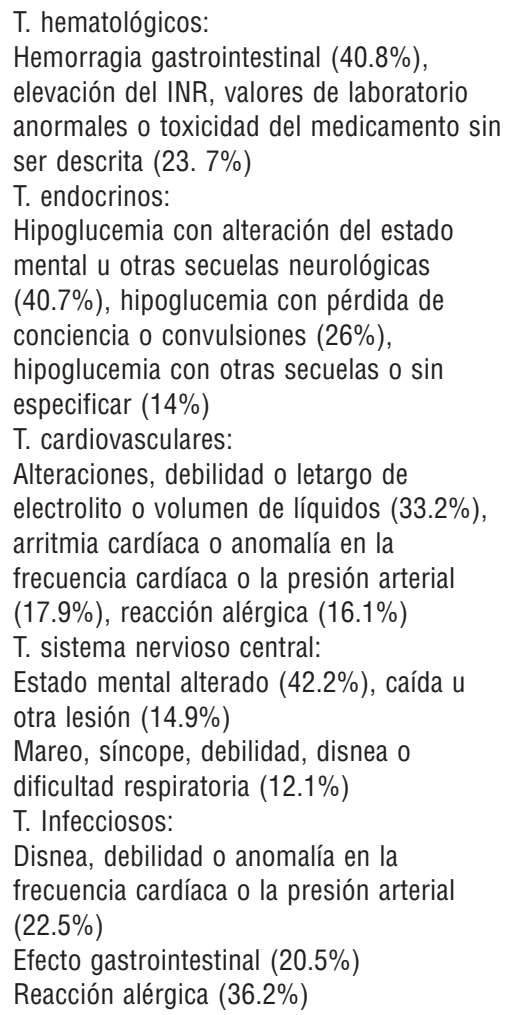 & $\begin{array}{l}\text { Warfarina }(33.3 \%) \\
\text { Insulinas }(13.9 \%) \\
\text { Agentes antiplaquetarios orales }(13.3 \%) \\
\text { Agente hipoglucemiantes orales }(10.7 \%)\end{array}$ \\
\hline
\end{tabular}

*Ingresos hospitalarios debidos a RAM.

†Reportadas en 22 de los 49 estudios.

†En los estudios incluidos en la publicación.

ACE: convertidores de la enzima angiotensina; AINE: antiinflamatorios no esteroideos; AT1: receptor angiotensina 1; EPOC: enfermedad pulmonar obstructiva crónica; INR: international normalized ratio; PT: término preferido; RAM: reacción adversa a medicamento; RAS: proteínas RAS; SOC: System Organ Class; T: trastornos.

Tomando en consideración el consolidado de la tabla previa, es posible observar una gran variedad de signos y síntomas no deseados (RAM) que se presentaron. Así mismo, se encontró amplia diversidad de medicamentos asociados a las RAM, en relación a los que, además, es posible observar múltiples formatos de clasificación, desde grupos terapéuticos hasta el listado del nombre del fármaco individual. Sin embargo, más allá de la heterogeneidad en el formato de reporte, fue notorio durante la revisión de los artículos que ninguno de los autores describió a los medicamentos utilizados en función de su marca comercial, ni tampoco se referenció el o los lotes de los medicamentos asociados a las RAM. Esta observación denota que el actual análisis de causalidad sigue estando enfocado a la molécula o fármaco, sin dar relevancia al resto de factores que también pueden causar la aparición de las RAM, como lo son las diferencias entre marcas o entre lotes de una misma marca, los que no se consideraron relevantes en el análisis de causalidad de las RAM de los artículos aquí revisados. Es de esperarse que futuras publicaciones comiencen a incluir estos y otros factores en los análisis de causalidad. 


\section{CONCLUSIÓN}

La detección oportuna y la evaluación de las RAM están siendo una prioritarias en las estrategias para mejorar la seguridad de los medicamentos. Como hemos podido documentar en esta revisión, el impacto que las RAM tienen en el ámbito hospitalario es relevante por su alta incidencia y evitabilidad. Esta revisión aporta evidencia de la necesidad del monitoreo continuo en cada centro de atención para la salud, así como de la importancia de una participación incluyente de todos los profesionales de la salud como factores clave para generar estrategias de manejo de las RAM que tengan impactos positivos en seguridad y sean clínicamente significativos.

\section{BIBLIOGRAFÍA}

1. Sharrar R, Dieck G. Monitoring product safety in the postmarketing environment. Ther Adv Drug Saf. 2013;4(5):211-9.

2. Ley General de Salud. Última reforma publicada en el Diario Oficial de la Federación, con fecha 21-06-2018.

3. Norma Oficial Mexicana NOM-220-SSA1-2016, Instalación y operación de la farmacovigilancia. Diario Oficial de la Federación, 2016.

4. Budnitz DS, Lovegrove MC, Shehab N, Richards CL. Emergency hospitalization for adverse drug events in older Americans. Surv Anesthesiol. 2011;365(21):2002-12.

5. Feinstein JA, Feudtner C, Kampe A. Adverse drug event-related emergency department visits associated with complex chronic conditions. Pediatrics. 2014;133(6):e1575-87

6. Varallo F, Capucho H, Planeta C, Mastroianni P. Possible adverse drug events leading to hospital admission in a Brazilian teaching hospital. Clinics. 2014;69(3):163-7.

7. Bénard LA, Miremont SG, Pérault PMC, Noize P, Haramburu F. Incidence of hospital admissions due to adverse drug reactions in France: The EMIR study. Fundam Clin Pharmacol. 2015;29(1):106-11.

8. Bouvy JC, De Bruin ML, Koopmanschap MA. Epidemiology of adverse drug reactions in Europe: A review of recent observational studies. Drug Saf. 2015;38(5):437-53.

9. Chan SL, Ang X, Sani LL, Ng HY, Winther MD, Liu JJ, et al. Prevalence and characteristics of adverse drug reactions at admission to hospital: a prospective observational study. Br J Clin Pharmacol. 2016:82:1636-46.

10. Cabré M, Elias L, Garcia M, Palomera E, Serra PM. Avoidable hospitalizations due to adverse drug reactions in an acute geriatric unit. Analysis of 3,292 patients. Med Clin (Barc). 2018;150(6):209-14.

11. Oscanoa TJ, Lizaraso F, Carvajal A. Hospital admissions due to adverse drug reactions in the elderly. A meta-analysis. Eur J Clin Pharmacol. 2017; 73(6):759-770
12. Rojas-Velandia $\mathrm{C}$ et al. Characterization of adverse drug reactions causing admission to an intensive care unit. Br J Clin Pharmacol 2017;83(5): 1134-40.

13. Ribeiro MR, Motta AA, Marcondes-Fonseca LA, Kalil-Filho J, Giavina-Bianchi P. Increase of $10 \%$ in the rate of adverse drug reactions for each drug administered in hospitalized patients. Clinics (Sao Paulo). 2018;73: e185.

14. Schurig AM, et al. Adverse drug reactions (ADR) and emergencies. Dtsch Arztebl Int. 2018;115(15):251-8.

15. Giardina C, et al. Adverse drug reactions in hospitalized patients: Results of the FORWARD (facilitation of reporting in hospital ward) study. Front Pharmacol. 2018;9(350):1-12.

16. Wadhwa T, El Sheikh SG, Rao PGM. Monitoring and reporting of adverse drug reactions due to cardiovascular drugs in patients admitted to a secondary care hospital in Northern Emirate- a prospective surveillance study. Indian J Pharm Pract. 2018;11(2):71-8.

17. Juntti PP, Neuvonen L. Drug-related deaths in a university central hospital. Eur J Clin Pharmacol. 2002:58:479-82.

18. Nair P, Chalmers L, Peterson GM, Bereznicki BJ, Castelino RL, Bereznicki LR. Hospitalization in older patients due to adverse drug reactions - The need for a prediction tool. Clin Interv Aging. 2016;11:497-505.

19. Sultana J, Cutroneo P, Trifirò G. Clinical and economic burden of adverse drug reactions. J Pharmacol Pharmacother. 2013;4(1):s73-7.

20. Patel TK, Patel PB. Mortality among patients due to adverse drug reactions that lead to hospitalization: a meta-analysis. Eur J Clin Pharmacol. 2018; 74(6):819-32.

21. Alhawassi TM, Krass I, Bajorek B, Pont LG: A systematic review of the prevalence and risk factors for adverse drug reactions in the elderly in the acute care setting. Clin Interv Aging. 2014;2079-86.

22. Price S, Holman C, Sanfilippo F, Emery J. Association between potentially inappropriate medications from the Beers criteria and the risk of unplanned hospitalization in elderly patients. Ann Pharmacother. 2014:48(1):6-16.

23. Onder $\mathrm{G}$, et al. Adverse drug reactions as cause of hospital admissions: results from the Italian Group of Pharmacoepidemiology in the Elderly (GIFA). J Am Geriatr Soc. 2002;50(12):1962-8.

24. Wu C, Bell M, Wodchis PW. Incidence and economic burden of adverse drug reactions among elderly patients in Ontario emergency departments. Drug Saf. 2012;35(9):769-81.

25. Nivya K, Sri SKV, Ragoo N, Jayaprakash B, Sekhar SM. Systemic review on drug related hospital admissions - A pubmed based search. Saudi Pharm J. 2015;23:1-8.

26. Wong W, et al. Assessment of costs associated with adverse events in patients with cancer. PLoS One. 2018;13(4):1-13.

27. Naranjo CA, et al. A method for estimating the probability of adverse drug reactions. Clin Pharmacol Ther. 1981;30(2):239-45.

28. Hallas J, et al. Drug related hospital admissions: The role of definitions and intensity of data collection, and the possibility of prevention. J Intern Med. 1990;228(2):1.

29. Brown EG, Wood L, Wood S. The Medical Dictionary for Regulatory Activities (MedDRA). Drug Saf. 1999;20(2):1-2.

30. European Medicines Agency. Guideline on good pharmacovigilance practices (GVP). Annex I - Definitions (Rev 4). Heads Med Agencies; 2017.

31. Carlson ME, Pompei P, Ales KL, MacKenzie CL. A new method of classifying prognostic comorbidity in longitudinal studies: Development and validation. J Chronic Dis. 1987;40:373-83.

32. Quan $\mathrm{H}$, et al. Coding algorithms for defining comorbidities in ICD-9-CM and ICD-10 administrative data. Med Care. 2005:43:1130-9.

33. Hartwig S, Siegel J, Schneider P. Preventability and severity assessment in reporting adverse drug reactions. Am J Heal Syst Pharm. 1992;49: 2229-32. 